\title{
Research Paper \\ Relationship Between Styles of Attachment to God and Death Anxiety Resilience in the Elderly

Leila Bitarafan ${ }^{1}$, Mahmoud Kazemi ${ }^{1},{ }^{*}$ Majid Yousefi Afrashte ${ }^{1}$

1. Department of Psychology, Faculty of Humanities, University of Zanjan, Zanjan, Iran.

Use your device to scan
and readthe artice online

Received: 04 Jun. 2017

Accepted: 19 Sep. 2017
Key words: Attachment to God, Resilience, Death anxiety, Elderly.

\begin{abstract}
A B S T R A C T
Objectives In modern societies, the elderly belong to the growing social groups that are becoming increasingly important due to the age pyramid. This research has been done to consider the simple and multiple relationships between the styles of attachment to God and resilience with dead anxiety in the elderly people in Zanjan City, Iran.

Methods \& Materials This study was a descriptive and correlational study. It included 242 elderly individuals aged 55 to 65 years and living in Zanjan during 2016-2017 using available sampling method. The Templer Death Anxiety Scale, Conner and Davidson Resilience Scale, and Beck and McDonald's Attachment to God questionnaires were distributed to all the selected participants. Finally, 231 data were considered for this study as 11 questionnaires were excluded due to lack of full cooperation. To analyze the data, Pearson correlation coefficient and multiple regression analysis were used.

Results The correlation of death anxiety with attention to God was -0.56 , trust against mistrust was -0.65 , trust in God was -0.53 , and relationship with God was -0.68 . The correlation between total attachment to God and death anxiety was -0.68 and between resiliency and death anxiety was -0.54 . All the correlations were significant at the level of 0.01 . Also, multiple regression analysis showed that predictor variables explained $64 \%$ of variance in death anxiety.

Conclusion Results indicated that the attachment to God and resiliency have a significant and negative correlation with death anxiety. Thus, it is recommended that the relevant authorities should consider training and skills associated with resilience and belief in God in old age and elderly care centers.
\end{abstract}

\section{Extended Abstract}

\section{Objectives}

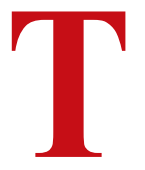

he elderly belonged to the growing social groups that have become increasingly important with regard to the population pyramid. Aging is a process associated with profound transformations in physiological, psychological and sociological dimensions of humans [1]. One of the important areas of health in the elderly is the psychological dimension that requires special attention so as to prevent disorders such as anxiety [2]. Anxiety is one of the most common problems of aging because this period is associated with feelings of deficiency and disability [3]. In such a situation, attachment to God and resilience can play decisive role in prevention and reduction of psychological disorders. Therefore, considering the importance of death anxiety, especially its incidence during the aging period, the present study aimed to investigate the simple and multiple relationships between styles of attachment to

\footnotetext{
* Corresponding Author:

Majid Yousefi Afrashteh, PhD

Address: Department of Psychology, Faculty of Humanities, University of Zanjan, Zanjan, Iran.

Tel: +98 (24) 33054450

E-mail: mjduosefi@gmail.com
} 
God and resilience with death anxiety in the elderly people in Zanjan City.

\section{Methods \& Materials}

The present study is a correlational-descriptive (regression analysis) research in which the variables of resilience and attachment to God were considered as predictors and the death anxiety as the criterion variable. The study population included all older adults in Zanjan City in 2016-2017 who were aged over 65 years. A total of 242 people were selected by available sampling method, and the purpose of the study was explained to them. After obtaining informed consent, the questionnaires were distributed among the participants. Of them, 11 questionnaires were excluded due to incomplete cooperation and misunderstanding of the questionnaire, and the data of 231 subjects were analyzed. The paper was approved by the Ethics Committee of the University of Zanjan.

The ethical considerations considered in this study included the following topics: 1 . the research participants were assured that their information would be preserved; and 2. the score was provided to those who would like to be informed. The tools used in this study were as follows: Templer death anxiety scale, Beck and McDonald's Attachment to God Inventory, and Connor-Davidson Resilience Scale. Finally, the data collected were analyzed by Pearson correlation coefficient and multiple regression analysis using SPSS18.

\section{Results}

Based on the descriptive findings of the study, attention to God had the highest mean (21.07) and standard deviation (8.51), while trust in God had the lowest mean (14.36) and standard deviation (3.47). Also, the means and standard deviations of the total attachment to God were 70.93 and 19.28 , total resilience were
53.94 and 16.48 , and death anxiety were 9.78 and 3.27 , respectively.

Table 1 shows the simple correlation coefficient between attachment to God, resilience and death anxiety. As shown in Table 1, all predictors have a significant negative relationship with death anxiety. The correlation between attention to God and death anxiety was -0.56 , between trust versus distrust and death anxiety -0.65 , between trust in God and death anxiety -0.53 , between relationship with God and death anxiety -0.66 , between total attachment to God and death anxiety -0.61 , and finally between resilience and death anxiety was -0.51 ; all of which are significant at the level of 0.01 .

In order to investigate the predictive role of attachment to God and the resilience in the dependent variable, the stepwise regression was used. The results of this analysis are as follows: The findings indicate that the values of regression coefficients in terms of predictability are $\beta=-0.38(\mathrm{P}=0.01)$ for relationship with God, $\beta=-0.27(\mathrm{P}=0.01)$ for trust in $\operatorname{God}, \beta=-0.30$ $(\mathrm{P}=0.01)$ for trust against distrust, $\beta=-0.22(\mathrm{P}=0.01)$ for resilience, and $\beta=-0.28(\mathrm{P}=0.01)$ for attention to God $(-0.28)$. Also, about $64 \%$ of the variance of death anxiety has been explained by predictor variables.

\section{Conclusion}

The results showed that the higher the level of resilience and attachment to God in the elderly, the less is the death anxiety. Regarding the last stage of the psychosocial development of Ericsson, which relates to the age and focuses on the reflection of past activities, those who fail at this stage will feel that their lives have been lost and will regret the past. In this case, the person is facing a sense of frustration and disappointment. Those who are proud of their past achievements in life will have a sense of integrity and identity. These people are those who even feel wise in the face of death.

Table 1. Correlation coefficients of attachment to God, resilience with death anxiety

\begin{tabular}{|c|c|c|c|c|c|c|c|}
\hline & 1 & 2 & 3 & 4 & 5 & 6 & 7 \\
\hline Attention to God & 1 & & & & & & \\
\hline Trust versus distrust & $0.56 * *$ & 1 & & & & & \\
\hline Trust in God & $0.44^{* *}$ & $0.38 * *$ & 1 & & & & \\
\hline Relationship with God & $0.55^{* *}$ & $0.71 * *$ & $0.37^{* *}$ & 1 & & & \\
\hline Total attachment to God & $0.78^{* *}$ & $0.83 * *$ & $0.52 * *$ & $0.81^{* *}$ & 1 & & \\
\hline Resilience & $0.26^{* *}$ & $0.48^{* *}$ & $0.27 * *$ & $0.54^{* *}$ & $0.50 * *$ & 1 & \\
\hline Death anxiety & $-0.56 * *$ & $-0.65 * *$ & $-0.53 * *$ & $-0.68 * *$ & -0.68 & $-0.54 * *$ & 1 \\
\hline
\end{tabular}


Therefore, they experience less death anxiety. According to the research findings, it is suggested to consider conducting training of necessary skills about resilience and belief in God for the elderly in elderly care centers.

\section{Acknowledgments}

This research did not receive any specific grant from funding agencies in the public, commercial, or not-forprofit sectors.

\section{Conflict of Interest}

The authors declared no conflicts of interest. 


\title{
رابطه سبكهاى دلبستَّى به خدا و تابآورى با اضطراب مركى در سالمندان
}

\author{
ليلا بيطرفان'، محمود كاظمى'، "مجيد يوسفى افراشته' \\ 1- كروه روانشئاسى، دانشكده علوم انسانى، دانشكاه زنجان، زنجان، ايران.
}

\begin{abstract}
حكSנ

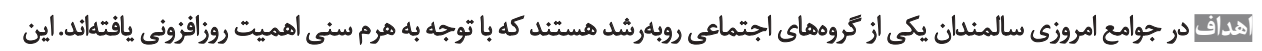

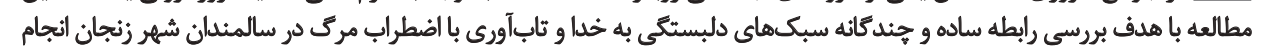
شده است.

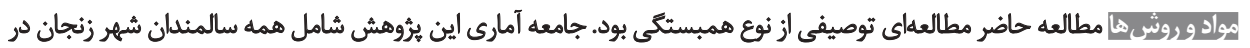

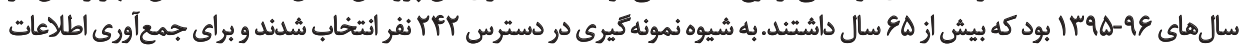

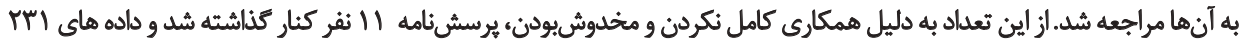

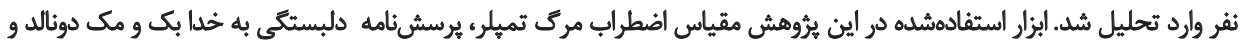

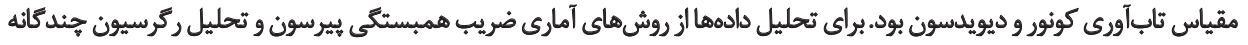

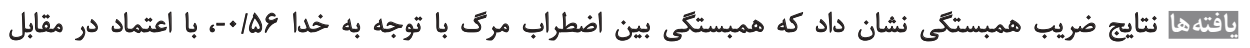

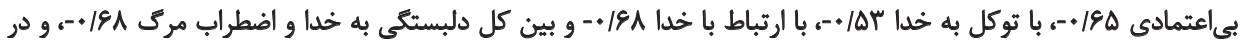

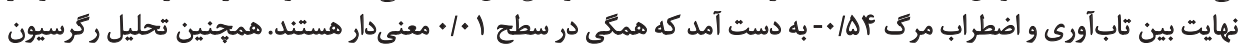

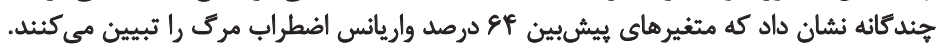

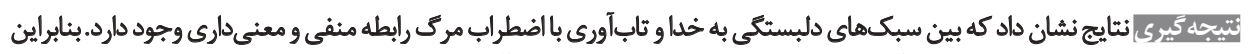

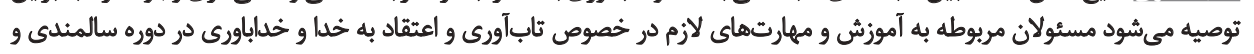

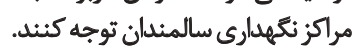

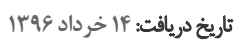

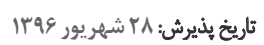

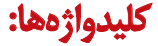

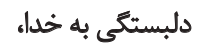

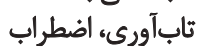

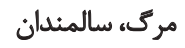

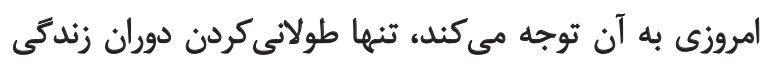

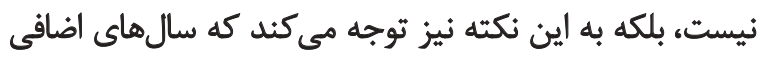

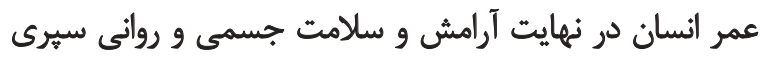

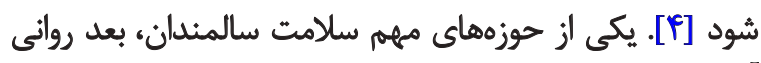

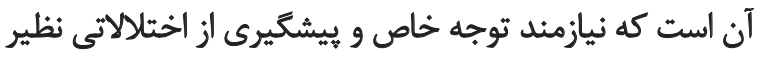

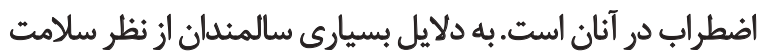

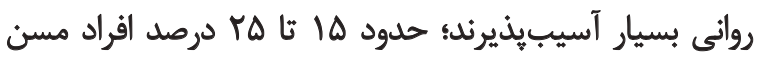
مشكلات روانشناختى مهمى دارند [ه]. اضطراب يكى از مسائل شايع دوره سالمندى است؛ زيرا اين ماني

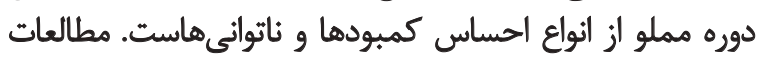

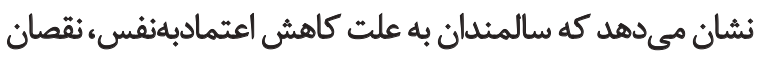

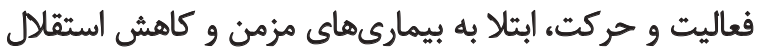

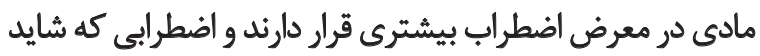

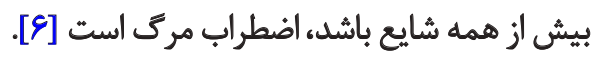

روند سالمندى در سالهاى اخير شتاب بيشترى گرفته است.

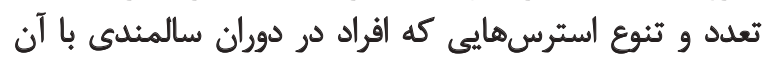

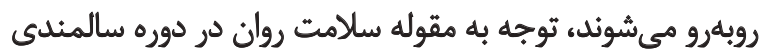

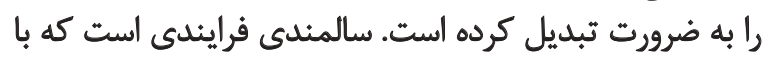

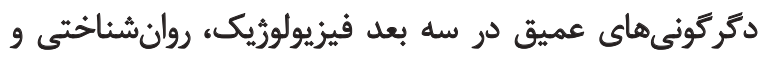

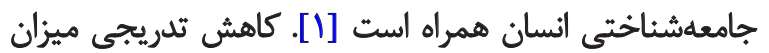

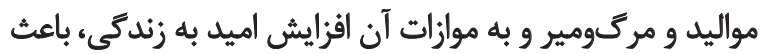

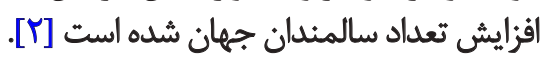

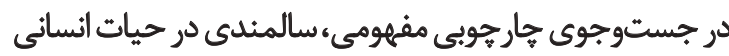

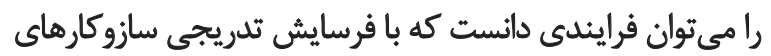

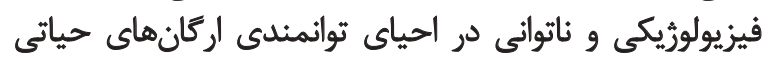

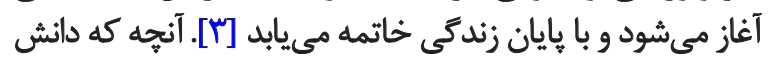




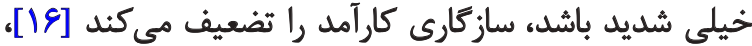

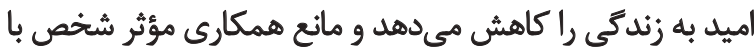

محيط مي أشود [IV

از طرفي تنوع و تعدد استرسهايى كه افراد در دوران سالمندى

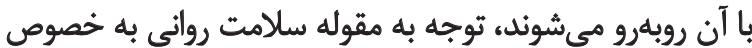

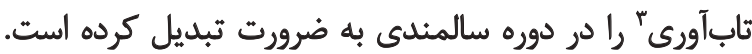

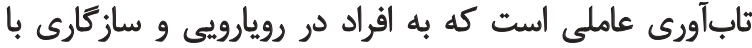

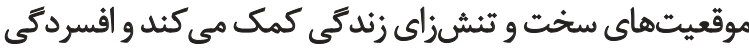

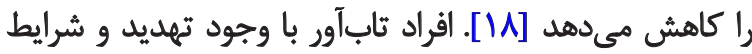

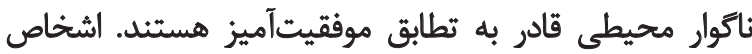

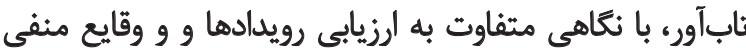

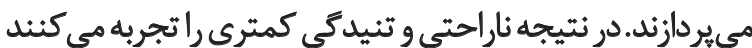

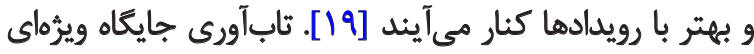

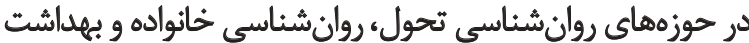

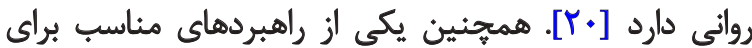

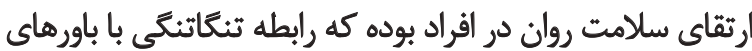

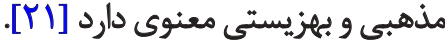

در حالىكه بسيارى از محققان در تعريف دقيق ثابآورى

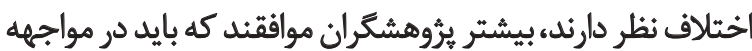

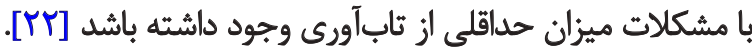

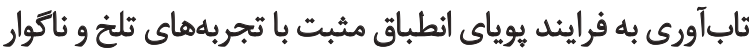

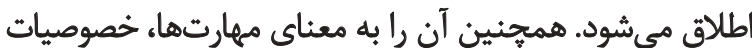

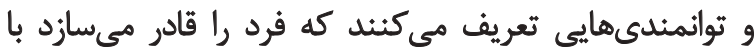

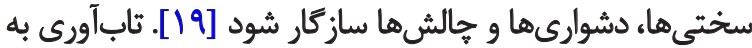

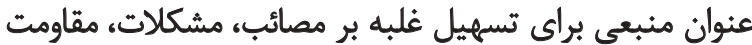

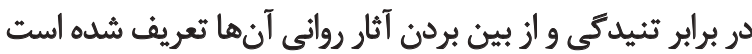

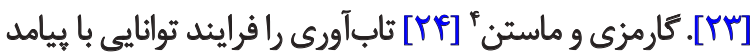

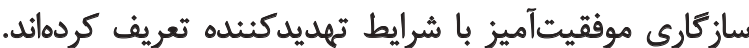

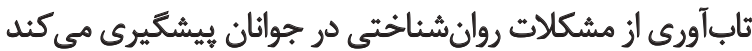

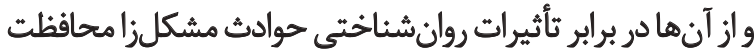

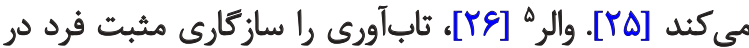
واكنش به شرايط ناكوار مي داند.

از ديرباز در روانشئاسى به موضوع مذهب توجه شده است

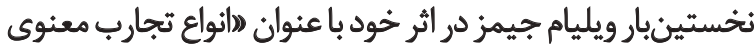

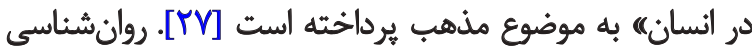

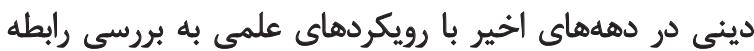

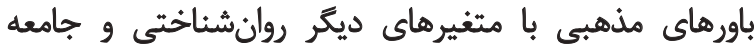

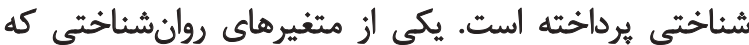

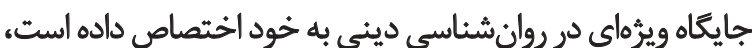

\footnotetext{
3. Resiliense

4. Garmezy \& Masten

5. Waller
}

اضطراب واكنش در برابر خطرى نامعلوم، دروني، مبهم و أز منشأ.

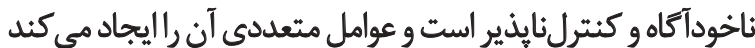

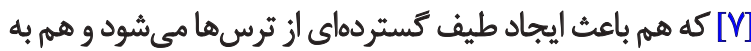

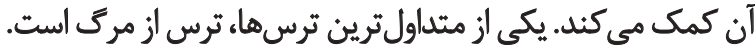

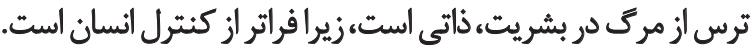

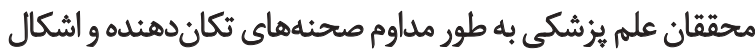

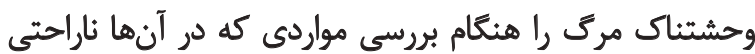

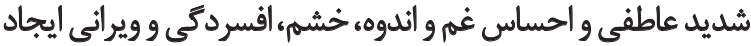

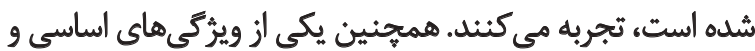

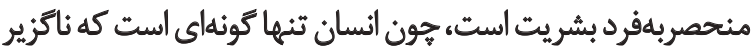

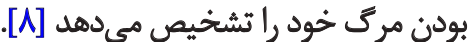
برخى از مشكلات اضطراب درواقع خودبيهخود، ترس از مرى

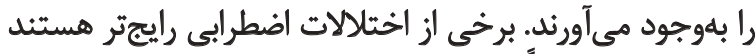

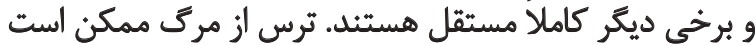

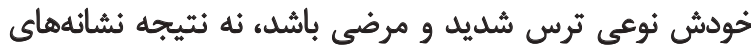

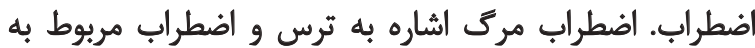

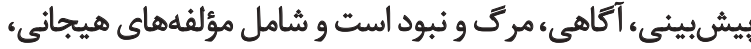

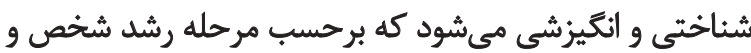

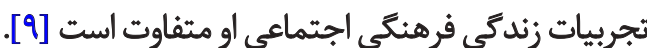

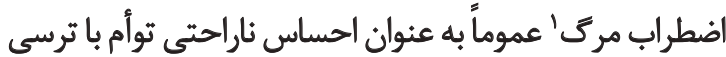

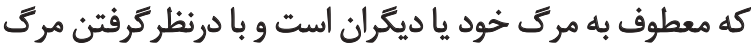

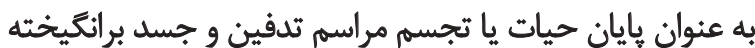

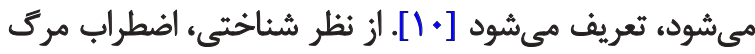

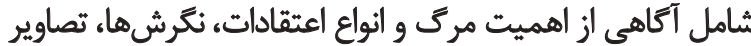

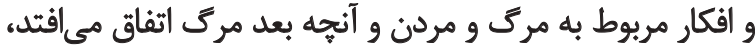

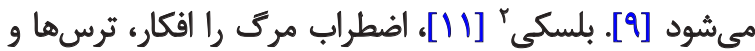

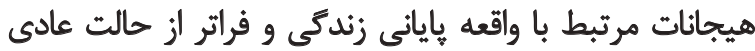

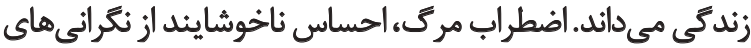

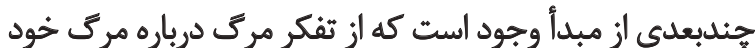

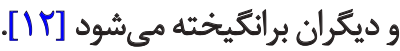

اضطراب مرى با ساختارهاى اساسى مغز در ارتباط است،

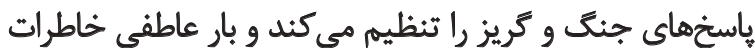

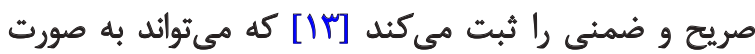

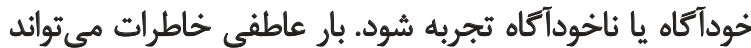

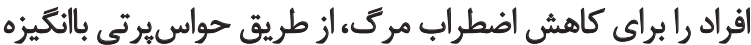

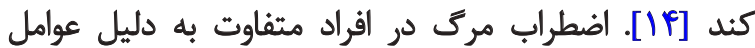

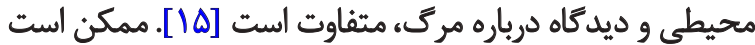

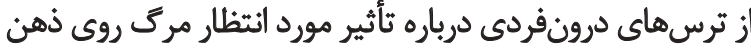

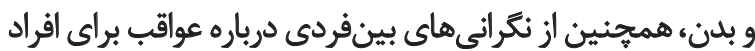

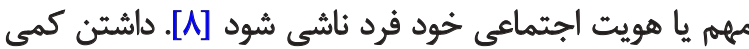

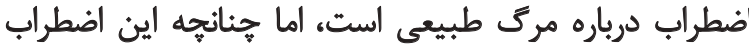

1. Death anxiety

2.Belskey 


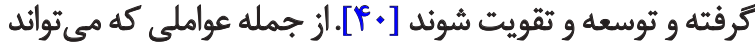

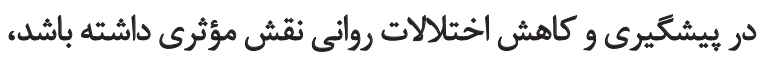

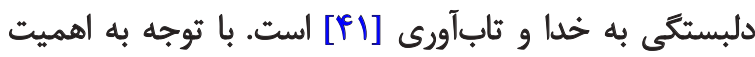

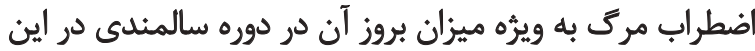

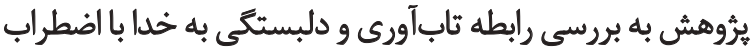
مركى در سالمندان برداخته شده است.

\section{ورش مطالعه}

روش يُروهش توصيفى از نوع همبستكى (تحليل ركرسيون)

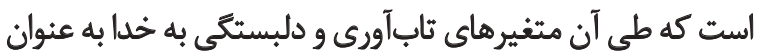

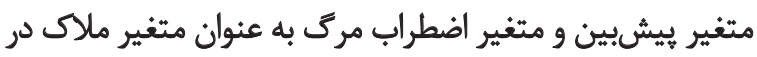

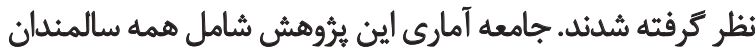

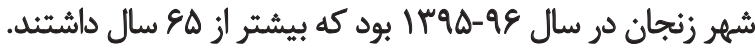

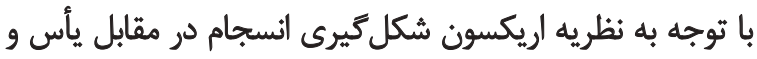

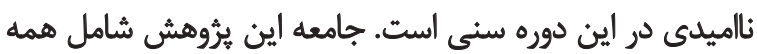

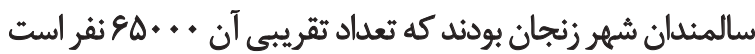

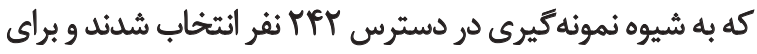

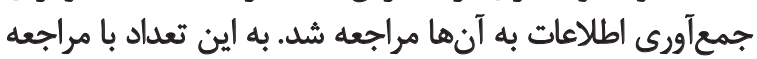

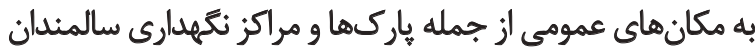

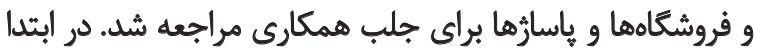

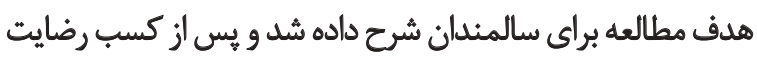

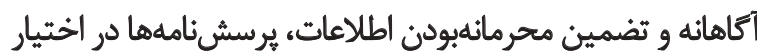

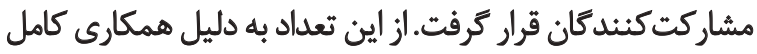

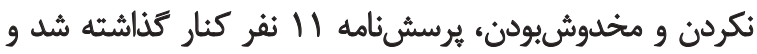

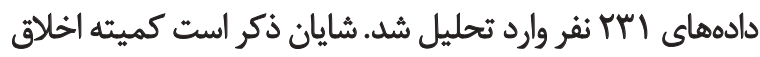
دانشكاه زنجان اين مقاله را تأييد كرده است.

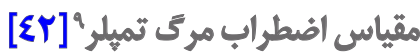

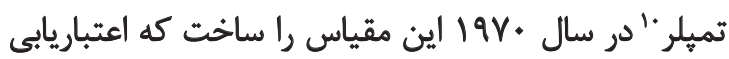

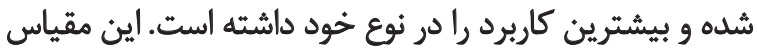

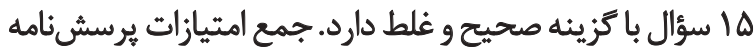

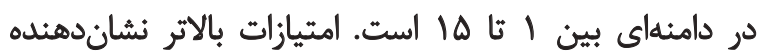

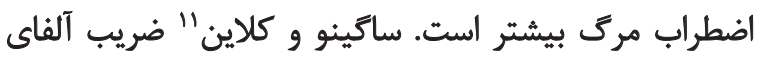

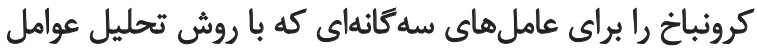

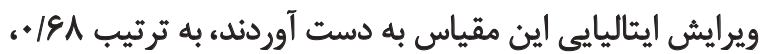

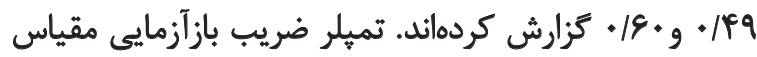

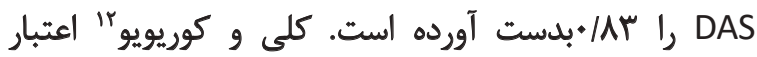

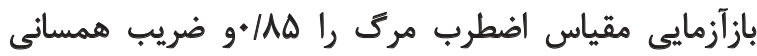

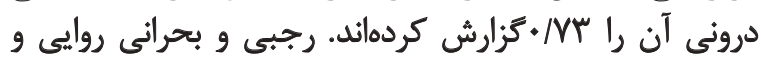

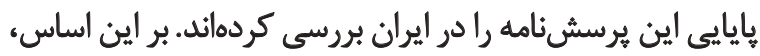

9. Death Anxiety Scale

10. Templer

11. Saggino \& Kline

12. Kelly \& Corriveau
دلبستكى به خداوند أست.نظريه دلبستكى نقش تعيين كنينداي

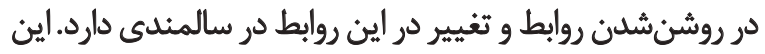

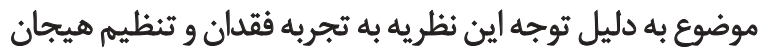

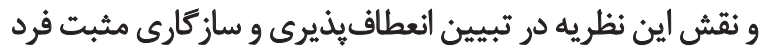

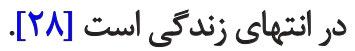

به طور كلى نظريه دلبستئي در دوران سالمندي بر جهمار حوزه

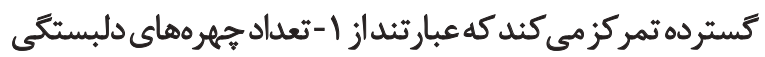

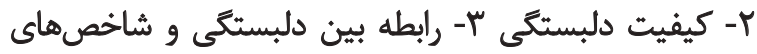

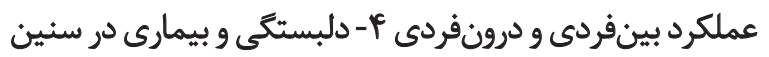

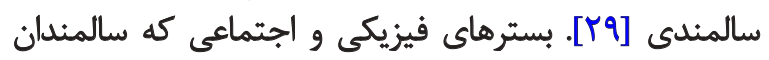

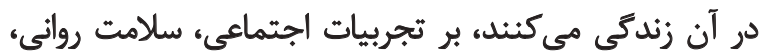

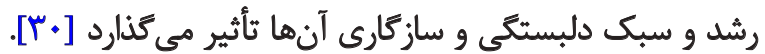

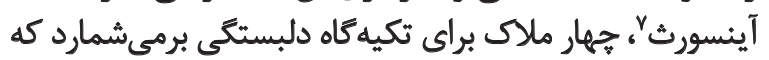

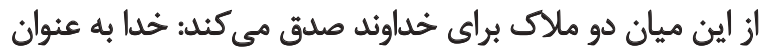

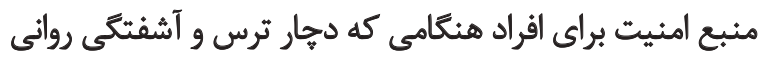

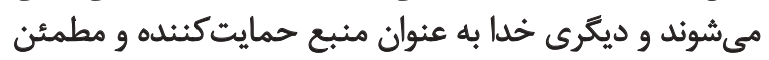

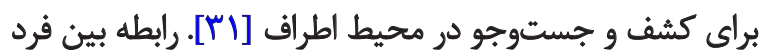

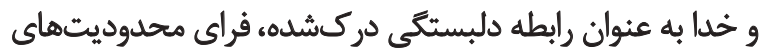

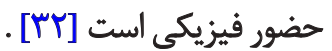

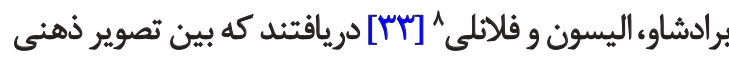

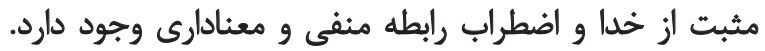

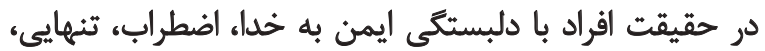

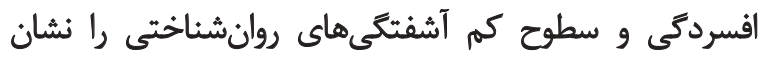

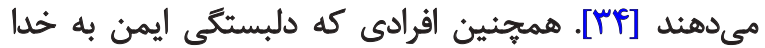

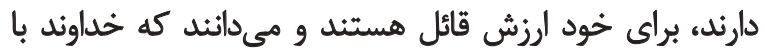

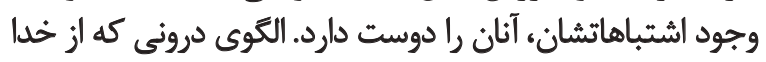

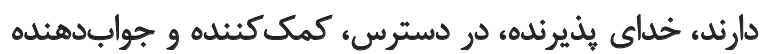

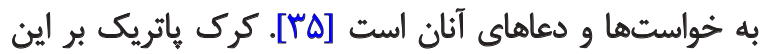

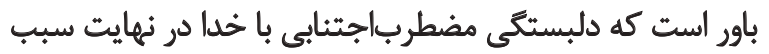

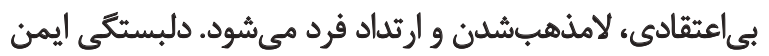

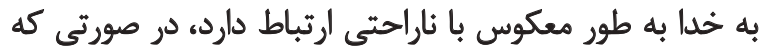

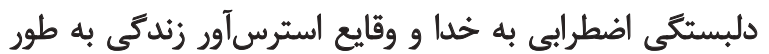

مثبت با ناراحتى ارتباط دارد [عبــ در كل تحقيقات نشان مى دهند كه دلبستكى ايمن باتوانمندى

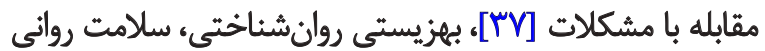

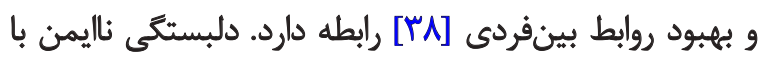

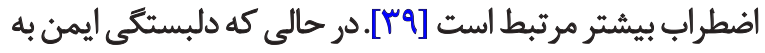

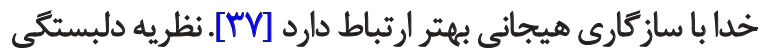

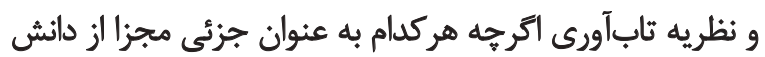

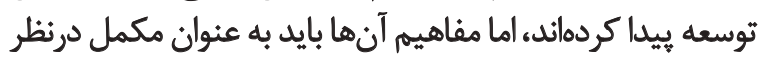
6. Attachment to God
7. Ainsworth
8. Bradshaw, Ellison \& Flannelly 
يافتهها

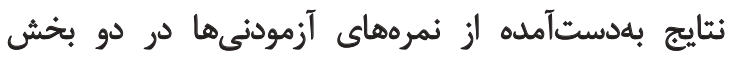

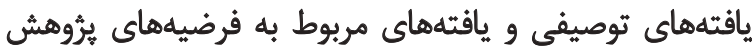

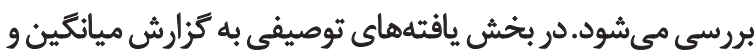

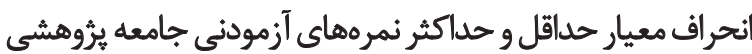

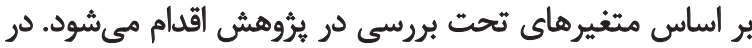

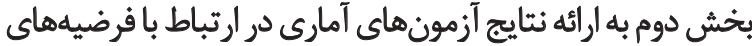

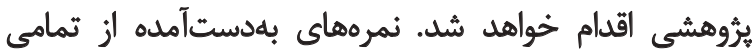

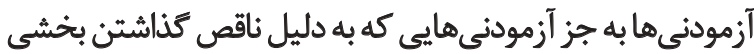

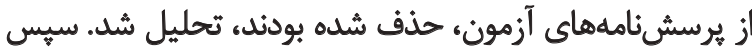

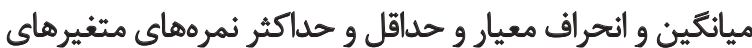

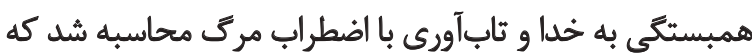

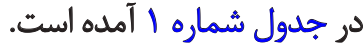

در مورد "جنسيت) از مجموع اسب نفر، وسا نفر (•ود درصد)

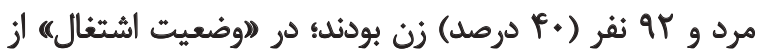

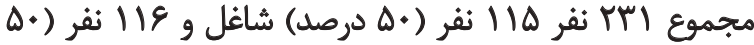

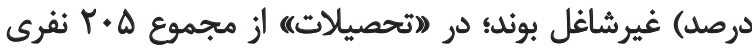

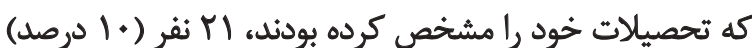

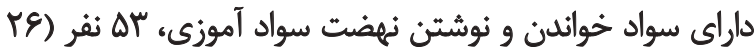

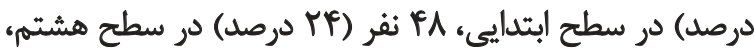

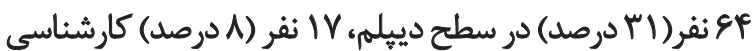

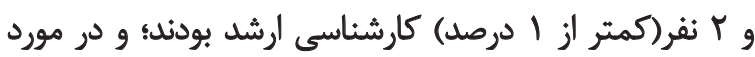

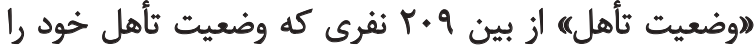

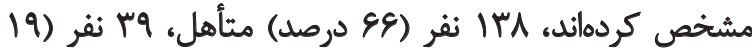

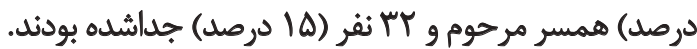
همانطور كه در جدول شماره آ آمده است، از بين متغيرهاى

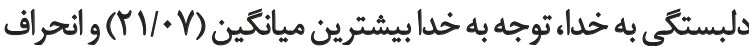

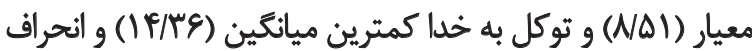

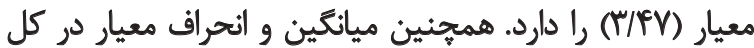

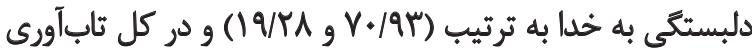

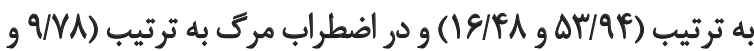

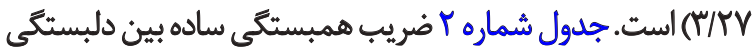
به خدا، تابآورى و اضطراب مرك رانشان انشان مى دهد.

همانطور كه در جدول شماره r مشاهده ميشودي، همه

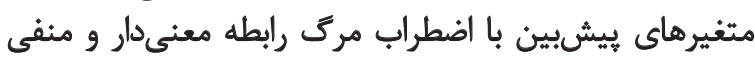

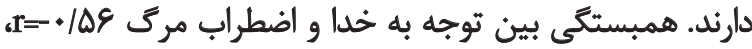

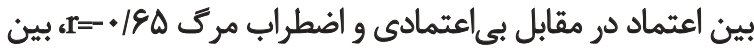

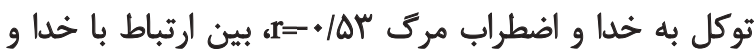

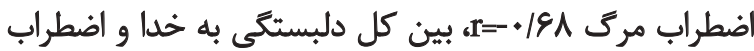

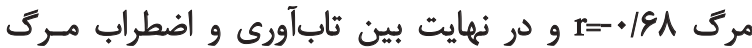

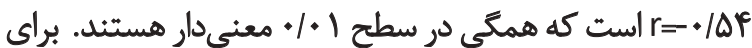

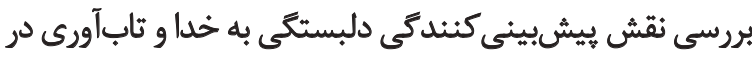

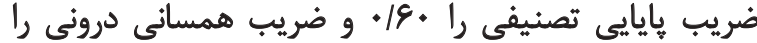

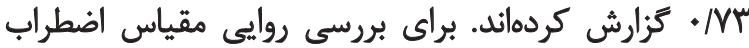

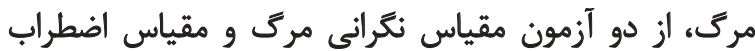

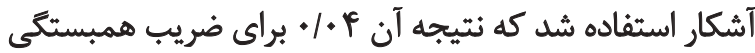

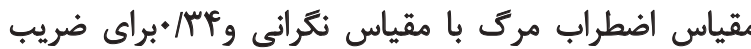

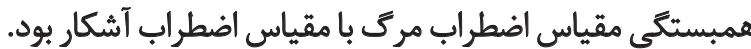

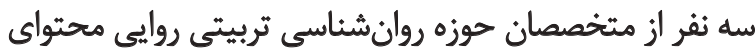

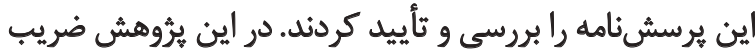

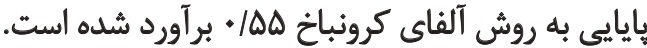

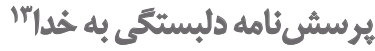
اين مقياس Y Y گويه دارد كه بك و مك دونالد آن را در

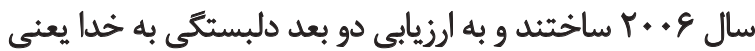

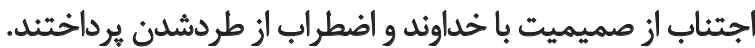

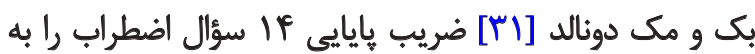

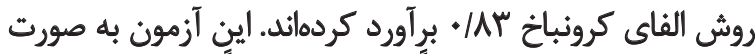

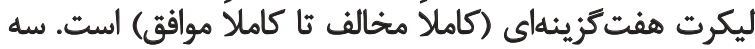

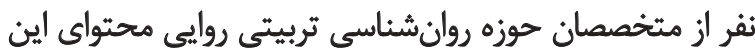

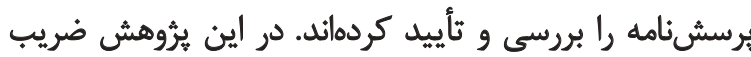

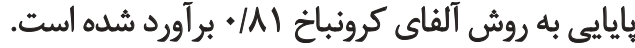

\section{مقياس تابآورى كوثور و ديويدسون (CD-RTS9)}

براى سنجش ثابآورى، مقياس ثابآورى كانر و ديويدسون

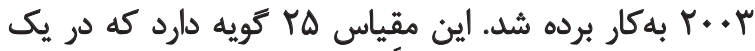

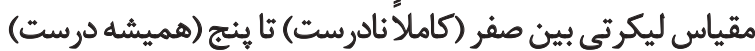

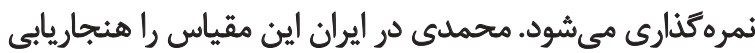

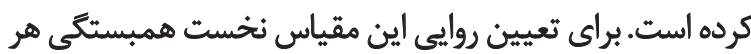

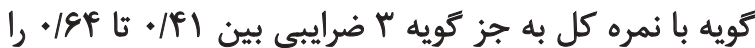

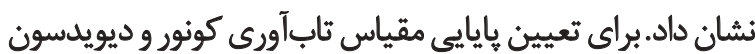

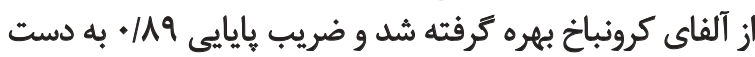

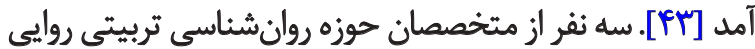

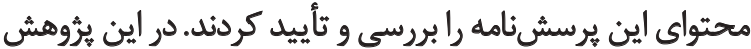

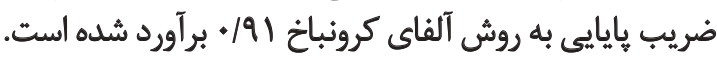
ملاحظات اخلاقي اين مطالعه، اين موضوعات را دربرداشت:

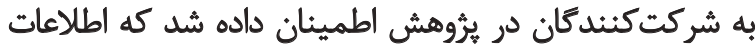

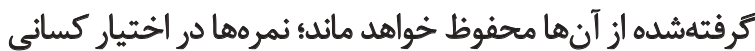

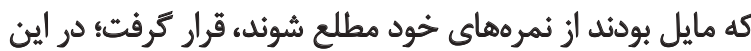

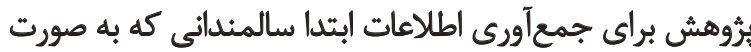

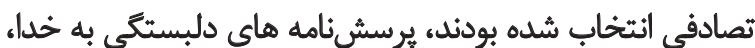

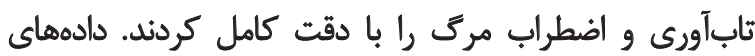

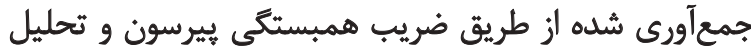

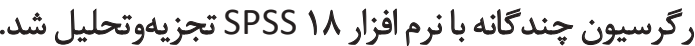

13. Attachment to God inventory 
جدول ا. ميانكين، انحراف معيار، حداقل و حداكثر نمرههاي آزمودنى ها در خردهمياس هاي دلبستّى به خدا و تاب آورى و اضطراب مرى

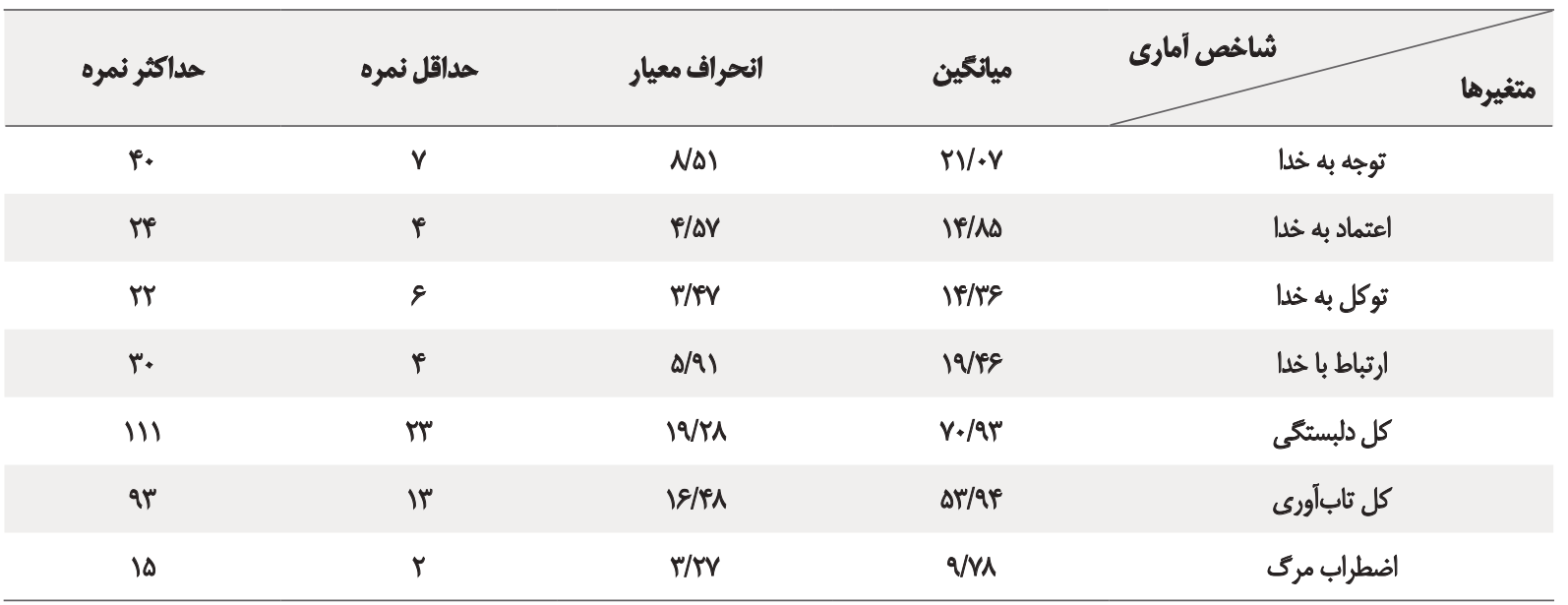

\begin{tabular}{ccccc}
\hline & & \\
\hline \\
\hline
\end{tabular}

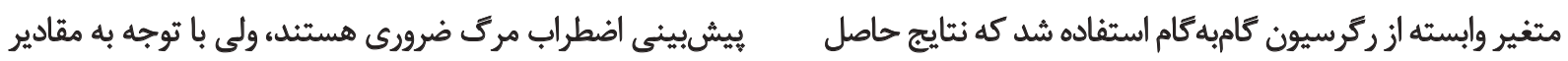

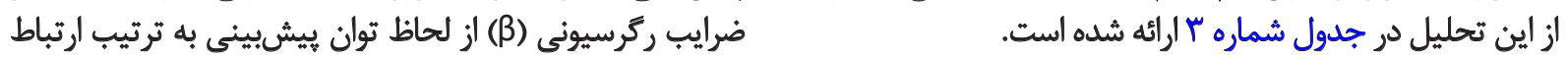

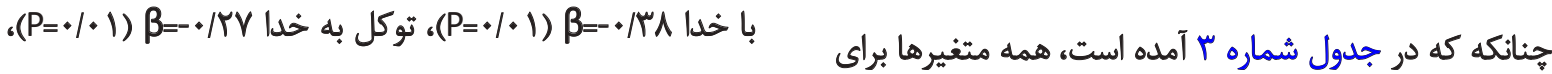

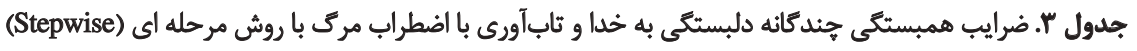

\begin{tabular}{|c|c|c|c|c|c|c|c|}
\hline $\mathbf{R}^{Y}$ & $F$ & Sig. & $\mathrm{t}$ & B & ضريب بثا & مقدار ثابت & شاخص آمارى \\
\hline \multirow[t]{6}{*}{ tef } & $9 \Delta / M$ & $4+\infty$ & $-\Delta / r+$ & $-d / r+$ & $-\oplus / \mathrm{M \Lambda}$ & MIIS & ارثباط با خدا \\
\hline & & $\%$ & $-\Delta / \Delta q$ &.$- / T \Delta$ &.$- / T V$ & & توكل به خدا \\
\hline & & .10. & $-r / u$ &.$- / \pi r$ & $-* / \pi$ & & اعتماد در برابر بي عتمادى \\
\hline & & 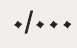 & $-r / / q$ & $-.1+\varphi$ &.$- / M r$ & & ثابآورى \\
\hline & & 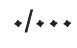 & $-r / a r$ &.-+111 &.$- / 14 \lambda$ & & توجه به حلا \\
\hline & & .10 & r/gA & -.1 .8 & $\cdot / m e$ & & كل دلبستكي به خدا \\
\hline
\end{tabular}


دورى از از خداوند است، رنج مىبرند.

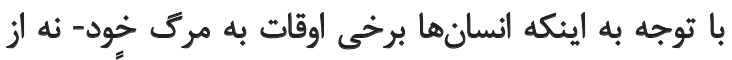

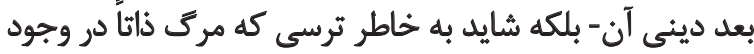

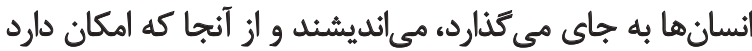

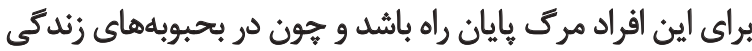

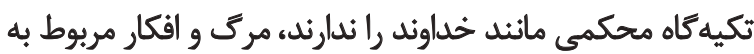

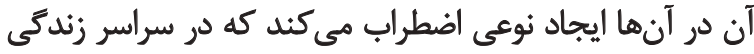

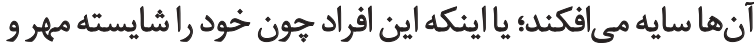

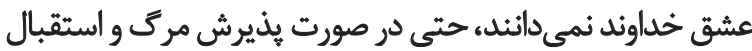

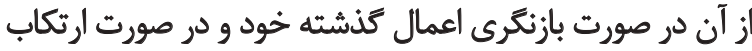

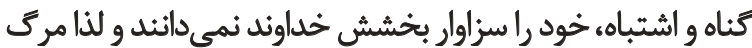

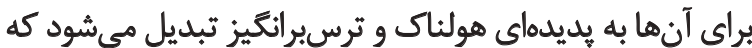

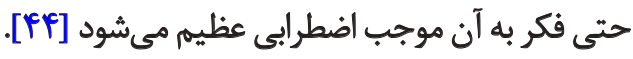

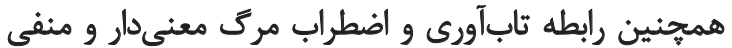

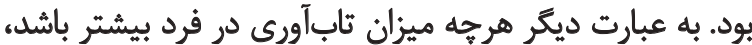

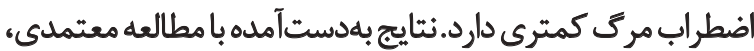
يروهىنيا و فاطمى اردستانى همسو است. در تبيين اين يافتهانها

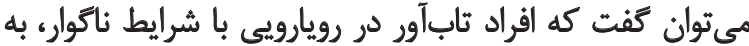

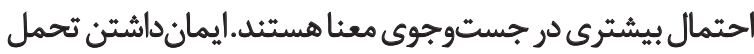

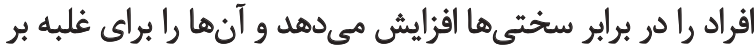

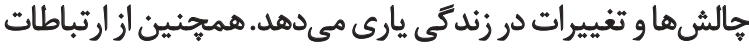

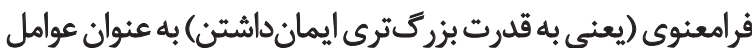

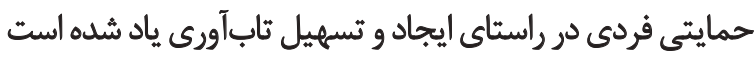

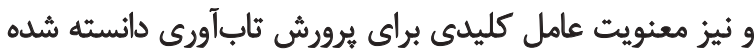

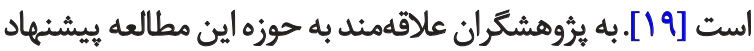

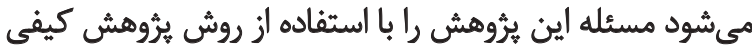
انجام دهند تانتايج به صورت مقايسه الى مكمل همث قرار كيرند.

\section{نتيجلكيرى نهايى}

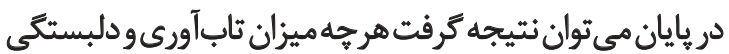

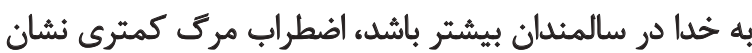

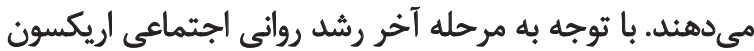

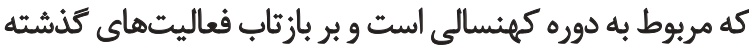

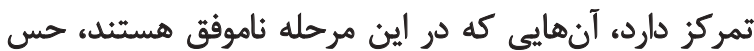

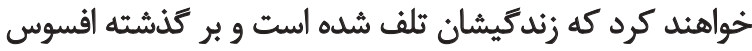

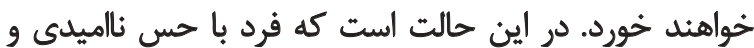

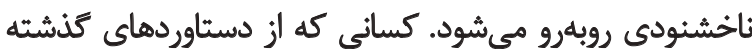

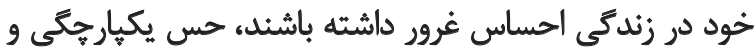

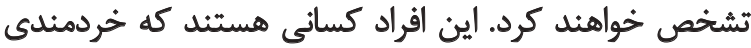

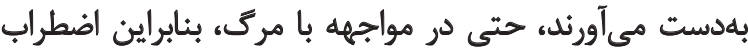
مركى كمترى تجربه مي كنيند. از اين رو بيشنهاد هي شود ثا در مراكز نكّهبارى سالمندان،

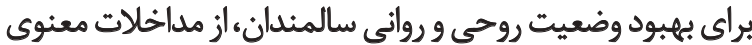

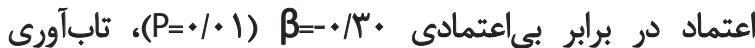
بو

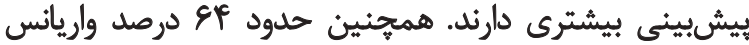

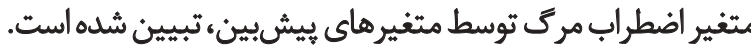

ثب

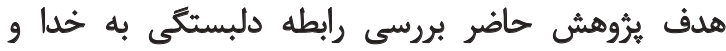

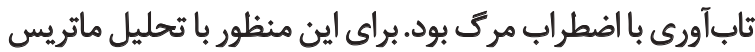

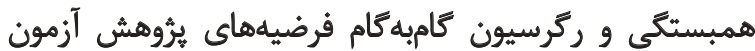

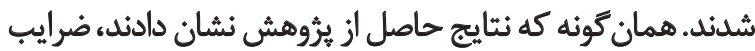

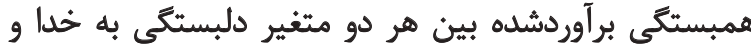

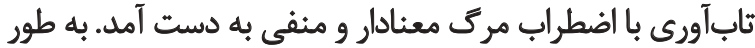

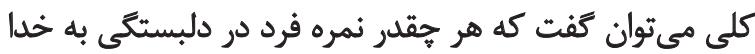

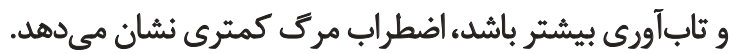
نتايج به دست آمده مبنى بر رابطه دلبستى بهى به خدا و اضطراب

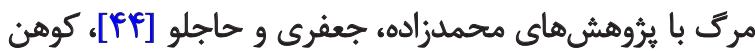

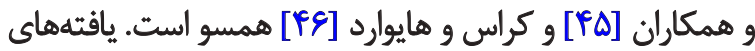

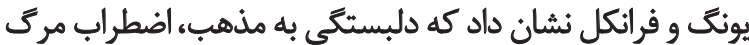

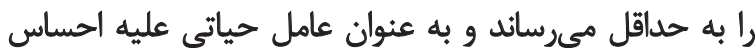

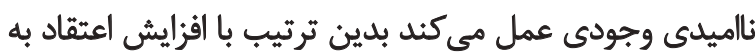

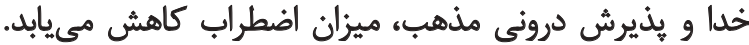

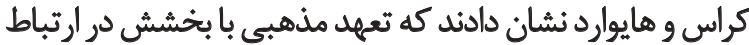

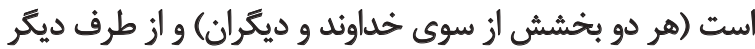
بخشش با كاهش اضطراب مرى همراه است.

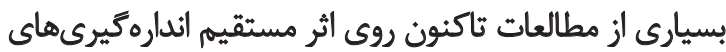

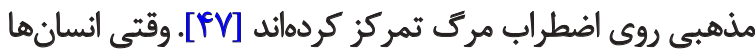

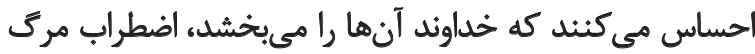

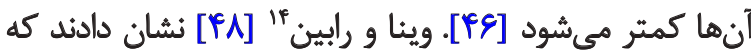

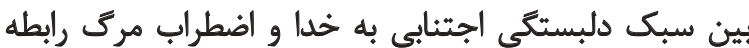

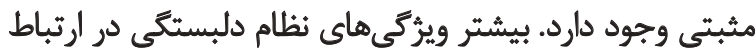

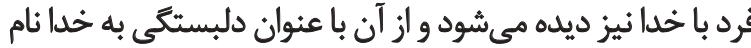

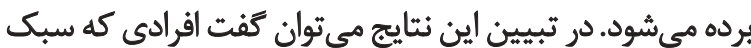

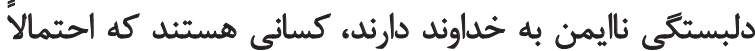

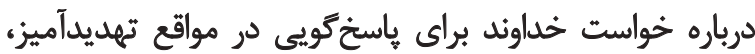

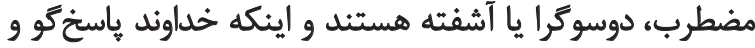

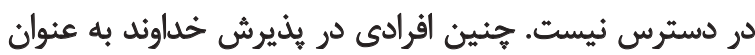

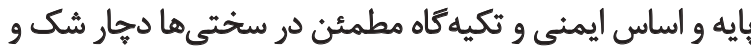

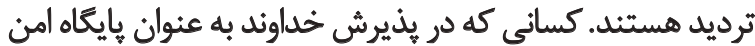

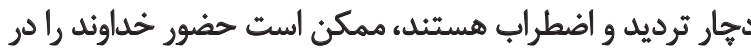

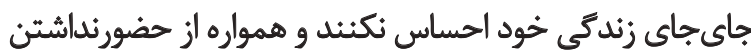

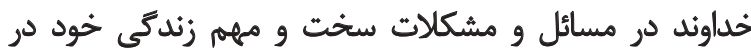
اضطراب باشند. جينين افرادى هميشه از نوعى اضطراب كه نشات نهانه

14. Viana \& Rabian 
و مذهبى استفاده شود. همجينين با توجه به آموختنىبودن آبوري

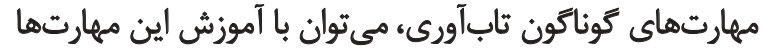

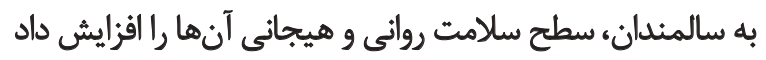
و ميزان اضطراب مرى را به كمترين حد ممكن رساند.

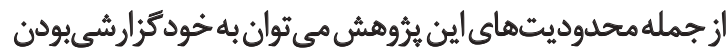

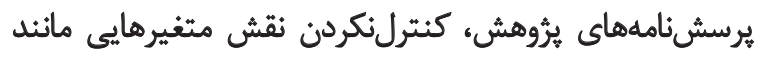

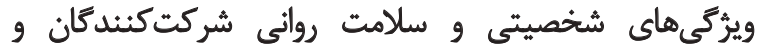
تعميمنايذيرى يرؤهش به ديكر كروهاي سالمندان اشاره كرد.

$$
\text { تشكر و قدردافي }
$$

در خايان، از همكارى مسئولان و كاركنان آسايشعادهاى شهر

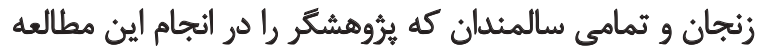

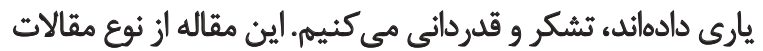
يُؤهشى است و حامى مالى ندارد. 


\section{References}

[1] Ghorbanalipour M, Ismail A. [Determining the efficacy of logo therapy in death anxiety among the older adults (Persian)]. Farhang-e Moshavere va Ravan Darmani.. 2012; 3(9):53-68.

[2] Afkhami A, Keshavarz SA, Rahimi A, Jazayeri SA, Sadrzadeh, Y. [Nutritional status and associated non-dietary factors in the elderly living in nursing homes of Tehran and Shemiranat, 2004 (Persian)]. Payesh. 2008; 7(3):211-17.

[3] Seyed Mirzaei SM .[ Some observations on the dimensions of aging with a look at Japan's experience (Persian)]. Journal of Human Sciences. 2007; (53):201-22.

[4] Rosendahl E, Gustafson Y, Nordin E, Lundin-Olsson L, Nyberg L. A randomized controlled trial of fall prevention by a high-intensity functional exercise program for older people living in residential care facilities. Aging Clinical and Experimental Research. 2008; 20(1):67-75. doi: 10.1007/bf03324750

[5] Ingersoll B, Silverman A. Comparative group psychotherapy for the aged. The gerontologist. 1978; 18(2):201-6. doi: 10.1093/ geront/18.2.201

[6] Brody CM, Semel VG. Strategies for therapy with the elderly: Living with hope and meaning. New York: Springer Publishing Company; 2005.

[7] Ghasempour A, Soreh J, Seied Tazeh Kand M. [Predicting death anxiety on the basis of emotion cognitive regulation strategies (Persian)]. Knowledge and Research in Applied Psychology. 2012; 13(2):63-70

[8] Geurtsen LI. A multidimensional approach of death anxiety: physical health, gender and psychosocial correlates in a community sample and a clinical sample of Dutch elderly people. [MSc. thesis]. Utrecht: Utrecht University; 2010.

[9] Lehto RH, Stein KF. Death anxiety: An analysis of an evolving concept. Research and Theory for Nursing Practice. 2009; 23(1):23-41. doi: 10.1891/1541-6577.23.1.23

[10] Firestone R, Catlett J. Beyond death anxiety: Achieving life-affirming death awareness. New York: Springer Publishing Company; 2009.

[11] Belsky J. Developmental origins of attachment styles. Attachment \& Human Development. 2002; 4(2):166-70. doi: $10.1080 / 14616730210157510$

[12] Thiemann P, Quince T, Benson J, Wood D, Barclay S. Medical students' death anxiety: Severity and association with psychological health and attitudes toward palliative care. Journal of Pain and Symptom Management. 2015; 50(3):335-42. doi: 10.1016/j. jpainsymman.2015.03.014

[13] Barrett C. Death anxiety. In: Gellman M, Turner JR, editors. Encyclopedia of Behavioral Medicine. New York: Springer; 2013.

[14] Greenberg J, Pyszczynski T, Solomon S, Simon L, Breus M. Role of consciousness and accessibility of death-related thoughts in mortality salience effects. Journal of Personality and Social Psychology. 1994; 67(4):627-37. doi: 10.1037/0022-3514.67.4.627

[15] Yoo YS, Cho OH, Cha KS, Boo YJ. Factors influencing post-traumatic stress in Korean forensic science investigators. Asian Nursing Research. 2013; 7(3):136-41. doi: 10.1016/j.anr.2013.07.002

[16] Berk LE. Development through the lifespan [Y. Seyed Mohamadi. Persian Trans]. Tehran: Arasbaran; 2001.
[17] Pasha A, Amini S. [The effect of reality therapy on life expectancy and anxiety of martyrs' wives (Persian)]. Social Psychology. 2009; 3(9):37-50

[18] White B, Driver S, Warren AM. Resilience and indicators of adjustment during rehabilitation from a spinal cord injury. Rehabilitation Psychology. 2010; 55(1):23-32. doi: 10.1037/a0018451

[19] Moetamedi A, Pajouhinia S, Fatemi Ardestani M H. [The impact of spiritual wellbeing and resiliency in predicting death anxiety among elderly people in Tehran (Persian)]. Shefaye Khatam. 2015;3(2):19-26

[20] Campbell-Sills L, Cohan SL, Stein MB. Relationship of resilience to personality, coping, and psychiatric symptoms in young adults. Behaviour Research and Therapy. 2006; 44(4):585-99. doi: 10.1016/j.brat.2005.05.001

[21] Smith L, Webber R, DeFrain J. Spiritual well-being and its relationship to resilience in young people: A mixed methods case study. SAGE Open. 2013; 3(2):2158244013485582. doi: $10.1177 / 2158244013485582$

[22] Hooper LM. Individual and family resilience: Definitions, research, and frameworks relevant for all counselors. Alabama Counseling Association Journal. 2009; 35(1):19-26.

[23] Ciccheti D, Gramzy N. Milestone in the development of resilience. Development and Psychopathology. 1993; 5(04):497-502. doi: $10.1017 / \mathrm{s} 0954579400006118$

[24] Garmezy N, Masten AS. The protective role of competence indicators in children at risk: Perspectives on stress and coping In Cummings EM, Greene AL, Karraker KH, editors, Life-Span Developmental Psychology: Perspectives on Stress and Coping. Hillsdale, NJ: Lawrence Erlbaum Associates; 1991.

[25] Pinquart M. Moderating effects of dispositional resilience on associations between hassles and psychological distress. Journal of Applied Developmental Psychology. 2009; 30(1):53-60. doi: 10.1016/j.appdev.2008.10.005

[26] Waller MA. Resilience in ecosystemic context: Evolution of the concept. American Journal of Orthopsychiatry. 2001; 71(3):290-97. doi: 10.1037//0002-9432.71.3.290

[27] Spilka B, Hood RW, Hunsberger B, Gorsuch R. The psychology of religion: An empirical approach. New York: Guilford Press; 2009.

[28] Mikulincer M, Shaver PR. Attachment in adulthood: Structure, dynamics, and change. New York: Guilford Press; 2007.

[29] Saunders R, Jacobvitz D, Zaccagnino M, Beverung LM \& Hazen N. Pathways to earned-security: The role of alternative support figures. Attachment \& Human Development, 2011; 13(4): 403-20. doi: 10.1080/14616734.2011.584405

[30] Wenger G, Burholt V. Changes in levels of social isolation and loneliness among older people in a rural area: A twenty-year longitudinal study. Canadian Journal on Aging. 2004; 23(2):115-27. doi: $10.1353 /$ cja. 2004.0028

[31] Zahed-babelan A, Rezaei Jamaloei H, Sobhani-Herfati R. [An investigation of the relationship between attachment to God and resilience with meaningful life among students (Persian)]. Knowledge and Research in Applied Psychology. 2012; 13(49):75-85.

[32] Kirkpatrick LA, Shaver PR. Attachment theory and religion: Childhood attachments, religious beliefs, and conversion. Jour- 
nal for the Scientific Study of Religion. 1990; 29(3):315-34. doi: $10.2307 / 1386461$

[33] Bradshaw M, Ellison CG, Flannelly KJ. Prayer, God imagery, and symptoms of psychopathology. Journal for the Scientific Study of Religion. 2008; 47(4): 644-59. doi: 10.1111/j.14685906.2008.00432.x

[34] Bradshaw M, Ellison CG, Marcum JP. Attachment to God, images of God, and psychological distress in a nationwide sample of Presbyterians. International Journal for the Psychology of Religion. 2010; 20(2):130-47. doi: 10.1080/10508611003608049

[35] Kirkpatrick LA. Attachment, evolution, and the psychology of religion. New York: Guilford Press; 2004.

[36] Rowatt WC, Kirkpatrick LA. Two dimensions of attachment to God and their relation to affect, religiosity, and personality constructs. Journal for the Scientific Study of Religion. 2002; 41(4):637-651. doi: 10.1111/1468-5906.00143

[37] Belavich TG, Pargament KI. The role of attachment in predicting spiritual coping with a loved one in surgery. Journal of Adult Development. 2002; 9(1): 13-29. doi: 10.1023/a:1013873100466

[38] Crowell JA, Fraley RC, Shaver PR. Measurement of individual differences in adolescent and adult attachment. In: Shaver PR, Cassidy J, editors. Handbook of Attachment, Theory, Research, and Clinical Applications. New York: Guilford Press; 2016.

[39] Rowatt W, Kirkpatrick LA. Two dimensions of attachment to God and their relation to affect, religiosity, and personality constructs. Journal for the Scientific Study of Religion. 2002; 41(4):63751. doi: $10.1111 / 1468-5906.00143$

[40] Davydov DM, Stewart R, Ritchie K, Chaudieu I. Resilience and mental health. Clinical Psychology Review. 2010; 30(5):479-95. doi: 10.1016/j.cpr.2010.03.003

[41] Samani S, Joukar B, Sahragard N. [Resiliency, mental health and life satisfaction (Persian)]. Iranian Journal of Psychiatry \& Clinical Psychology . 2007; 13(3):290-295.

[42] Templer DI. The construction and validation of a death anxiety scale. The Journal of General Psychology. 1970; 82(2):165-77. doi: 10.1080/00221309.1970.9920634

[43] Mohamadi M. [Study on resiliency factors in people at risk for substance abuse (Persian)] [PhD Thesis]. Tehran: University of Social Welfare and Rehabilitation Sciences; 2006.

[44] Mohammadzade A, Jafari E, Hajlou N. [Relationships of death anxiety with religious coping, attachment to god and acting on religious beliefs (Persian)]. Culture in the Islamic University. 2015; 5(15):259-72.

[45] Cohen AB, Pierce JD, Chambers J, Meade R, Gorvine BJ, Koenig HG. Intrinsic and extrinsic religiosity, belief in the afterlife, death anxiety, and life satisfaction in young Catholics and Protestants. Journal of Research in Personality. 2005; 39(3):307-24. doi: 10.1016/j.jp. 2004.02.005

[46] Hayward RD, Krause N. Religion, mental health, and wellbeing: Social aspects. In: Saroglou V, editor. Religion, personality, and social behavior. London: Psychology Press; 2014.

[47] Harding SR, Flannelly KJ, Weaver AJ, Costa KG. The influence of religion on death anxiety and death acceptance. Mental Health, Religion \& Culture. 2005; 8(4):253-61. doi: 10.1080/13674670412331304311
[48] Viana AG, Rabian B. Perceived attachment: Relations to anxiety sensitivity, worry, and GAD symptoms. Behaviour Research and Therapy. 2008; 46(6):737-47. doi: 10.1016/j.brat.2008.03.002 